\title{
Prospective and randomised evaluation of the protease-modulating effect of oxidised regenerated cellulose/collagen matrix treatment in pressure sore ulcers
}

\author{
Oliver Kloeters ${ }^{1}$, Frank Unglaub ${ }^{2}$, Erik de Laat ${ }^{1}$, Marjolijn van Abeelen ${ }^{1}$ \& Dietmar Ulrich ${ }^{1}$ \\ 1 Department of Plastic and Reconstructive Surgery, Radboud University Hospital, Nijmegen, The Netherlands \\ 2 Department of Handsurgery, Vulpius Klinik, Bad Rappenau, Germany
}

\section{Key words}

Chronic wounds; Clinical trial; Elastase; Plasmin; Pressure sores; Proteases; Wound healing

\section{Correspondence to}

O Kloeters, MD, PhD

Department of Plastic and Reconstructive

Surgery

Radboud University Hospital

P.O. 9101

6500 HB Nijmegen

The Netherlands

E-mail: oliver.kloeters@radboudumc.nl

doi: 10.1111/iwj.12449
Kloeters O, Unglaub F, de Laat E, van Abeelen M, Ulrich D. Prospective and randomised evaluation of the protease-modulating effect of oxidised regenerated cellulose/collagen matrix treatment in pressure sore ulcers. Int Wound J 2016; 13:1231-1236

\section{Abstract}

In chronic wounds, excess levels and activity of proteases such as elastase and plasmin have been detected. Oxidised regenerated cellulose/collagen matrix (ORC/collagen matrix) has been reported to ameliorate the wound microenvironment by binding and inactivating excess proteases in wound exudates. In this study, the levels and activity of elastase and plasmin in wound exudates of pressure sore ulcers were measured to determine the beneficial effect of ORC/collagen matrix treatment compared with control treatment with a foam dressing. A total of 33 patients with pressure sores were enrolled in the study and were followed up for 12 weeks after treatment. Ten control patients were treated with a foam hydropolymer dressing (TIELLE ${ }^{\circledR}$, Systagenix), and the remaining 23 patients were treated with ORC/collagen matrix plus the foam dressing (TIELLE $^{\circledR}$, Systagenix) on top. Wound assessments were carried out over 12 weeks on a weekly basis, with dressing changes twice a week. Ulcers were photographed and wound exudates were collected on admission and at days 5, 14 and then every 14 days to provide a visual record of any changes in appearance of the ulcer and healing rate and for biochemical analysis of the wound. The levels and activity of elastase and plasmin were measured in wound exudates. Statistical analysis was performed using ANOVA and Bonferroni's post hoc test with $P$-values $<0.05$ considered to be significant. Compared with controls, ORC/collagen matrix - treated pressure sore wounds showed a significant faster healing rate, which positively correlated with a decreased activity of elastase and plasmin in wound exudates. No signs of infection or intolerance to the ORC/collagen matrix were observed.

\section{Introduction}

Chronic wounds are a heterogeneous group of skin lesions that do not progress through the normal healing process $(1,2)$. They include diabetic foot ulcers, vascular insufficiency ulcers at the lower extremities as well as pressure ulcers. In general, two consistent findings within the pathogenesis of these wounds are chronic inflammation and imbalance in protease and protease inhibitor $(1,2)$. Normal wound healing is a rigorously concerted remodelling process that consists of restorative activities (new tissue formation) as well as removal of debris. Within this complex environment, there are many points of regulation that precisely control the biological processes necessary to achieve normal wound repair (3). An alteration in any of these

\section{Key Messages}

- chronic wounds are characterised by a state of ongoing inflammation and overabundance of proteases resulting in the degradation of growth factors and fibronectin and reduction in the levels of endogenous protease inhibitors

- oxidised regenerated cellulose (ORC)/collagen matrix is known to reduce the levels and activities of destructive 
proteases such as elastase and plasmin in diabetic and venous leg ulcers

- ORC/collagen matrix reduces elastase and plasmin activity and enhances wound closure significantly in pressure sore ulcers

physiological processes can lead to a non-healing state and the formation of a chronic wound (4). These chronic wounds fail to follow the normal pattern of wound repair involving inflammation, granulation and reepithelialisation, and remain in a persistent inflammatory state. The latter is characterised by complement degradation and ongoing proteolysis, as indicated by increased levels of neutrophil elastase and gelatinases (5). The resulting degradation of matrix molecules, such as fibronectin, laminin and various collagens (5), hinders new tissue formation and cell adhesion, thereby preventing the constitution of proper contacts between keratinocyte integrins and underlying matrices in chronic wounds.

Elastase is one of the most destructive enzymes in the setting of wound healing and has been well characterised in non-healing wounds (6). Elastase possesses a broad specificity, preferentially cleaving bonds that are carboxy-terminal to valine and to a lesser extent alanine (7).

Plasmin is another serine protease whose primary substrate is fibrinogen/fibrin. It is converted from plasminogen through enzymatic cleavage by plasminogen activator, which also belongs to the family of serine proteases (1). Plasmin participates in a variety of pericellular proteolytic events, such as cell migration and angiogenesis (8). It activates matrix metalloproteinases (MMP) and growth factors such as transforming growth factor (TGF- $\beta$ ) $(9,10)$. Furthermore, plasmin plays an important role in vascular endothelial growth factor (VEGF) degradation, a well-described key cytokine in angiogenesis (11).

Interestingly, fibrin deposition is a common feature of non-healing wounds, such as venous leg ulcers, diabetic foot ulcers and pressure ulcers (12). However, an excessive concentration of both the serine protease elastase and plasmin in chronic non-healing wounds is to a significant degree responsible for the degradation of growth factors and fibronectin and reduction in the levels of endogenous inhibitors $(6,11,13)$.

ORC/collagen matrix, a device composed of oxidised regenerated cellulose and collagen, has been shown in previous studies to reduce the activity of elastase, plasmin and metalloproteinase in chronic wound fluids of diabetic patients in vitro (14-19). Moreover, in a randomised controlled study, ORC/collagen matrix accelerated the healing rate in venous leg ulcers compared with control wounds $(20,21)$.

In this pilot study, we sought to assess elastase and plasmin activity in wound fluids derived from pressure ulcers treated with ORC/collagen matrix compared with standard treatment. Furthermore, we clinically compared the healing rate of ORC/collagen matrix-treated wounds with control wounds.

\section{Patients and methods}

\section{Patients}

Patients were randomly assigned to the treatment or control group. Inclusion criteria were more than 18 years of age, chronic wounds present for at least 6 weeks, but $<12$ weeks prior to study enrolment, wound size more than $1 \mathrm{~cm}^{2}$ and debridement of wound before enrolment. Exclusion criteria were systemic inflammatory diseases, presence of malignant tumours, chemotherapy and alcohol and/or drug intoxication. In average, the Campbell score of the pressure ulcers was 3-4. All study procedures were carried out according to the ethical guidelines of the Declaration of Helsinki and were reviewed and approved by the local ethics committee. Individual informed consent was obtained from each patient.

Ten patients were randomly assigned to the control group. The wounds in this group were treated with an absorbing hydropolymer dressing (Tielle ${ }^{\circledR}$, Systagenix) alone. The ORC/collagen matrix group contained 23 patients, whose wounds were treated with the ORC/collagen and Tielle ${ }^{\circledR}$ on top. In both groups, dressing changes were carried out every 2-3 days. Wound fluids were collected from all 33 patients with pressure sores on admission, on days 5 and 14 and then every 2 weeks until week 12 . The majority of patients enrolled were elderly without significant differences in the age between both groups (average $63 \pm 8$ years).

\section{Chronic wound fluid collection and elution}

Wound fluid was collected by absorption onto a piece of RELEASE $^{\circledR}$ (Johnson \& Johnson Medical Ltd., Ascot, UK) dressing, which was placed directly on the pressure ulcer. The RELEASE $^{\circledR}$ dressing was cut to the size of the wound, placed in contact with the ulcer bed for 6 hours and covered with BIOCLUSIVE $^{\circledR}$ (Johnson \& Johnson Medical Ltd.). The dressing was then removed and frozen at $-80^{\circ} \mathrm{C}$ until elution of the wound fluid. Wound fluids from all the 33 patients with pressure sores were collected on days 0,5 and 14 and subsequently every 14 days thereafter for 12 weeks.

\section{Protein}

Total protein was determined according to the method of Bradford using a bovine serum albumin standard curve as control (22).

\section{Protease activity assay}

\section{Elastase assay}

The levels of elastase activity present in the wound fluid samples were measured spectroflourimetrically using substrate activity assays (Enzchek Elastase assay, Molecular Probes, Leiden, The Netherlands). The substrate comprises short peptides synthesised to mimic the appropriate enzyme cleavage site and contains a fluorescent group, which is released upon hydrolysis. Activity was expressed as either units per minute or corrected for total protein. Each sample was tested three times, and the average value was calculated. 


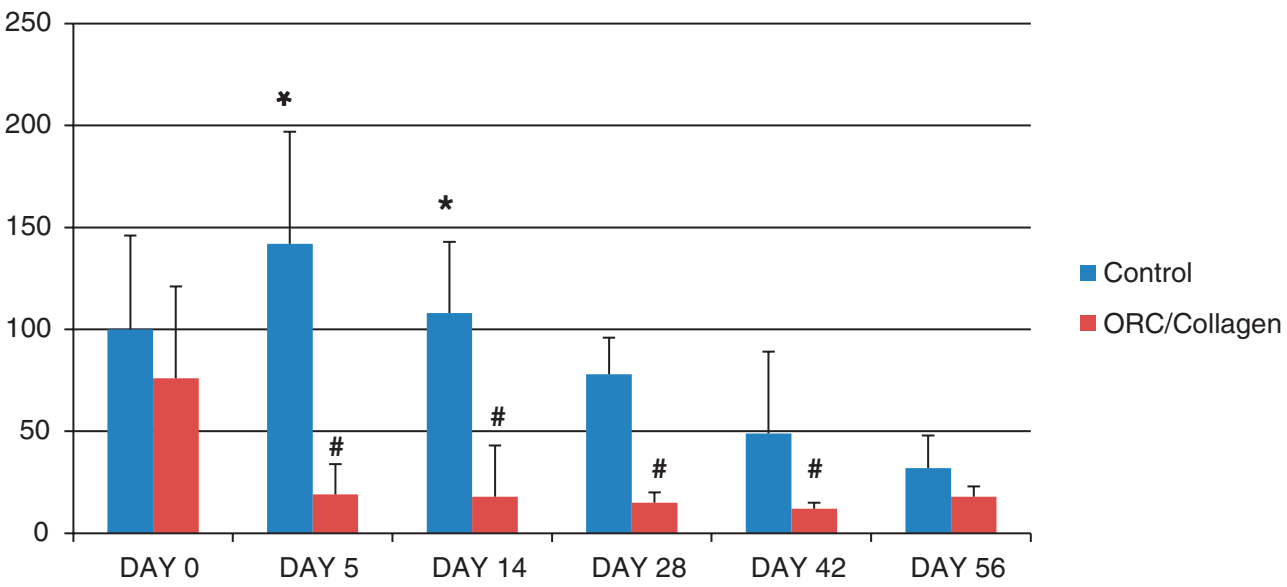

Figure 1 Elastase activity from day 0 to day 56 ( $y$-axis: $\mu \mathrm{U} / \mathrm{ml} / \mathrm{mg}$ protein). The elastase activity was significantly reduced in the oxidised regenerated cellulose (ORC)/collagen group on day 5 and day 14 compared with the control group $\left({ }^{*}\right)$. Furthermore, elastase activity was reduced on days $5,14,28$ and 42 compared with the baseline value at day 0 (\#), $(P<0.05, n=33)$.

The resulting increase in fluorescence was monitored with a fluorescence microplate reader. A selective inhibitor of elastase, $N$-methoxysuccinyl-Ala-Ala-Pro-Val-chloromethylletone, was used for confirming the identity of the protease responsible for substrate digestion.

\section{Plasmin activity}

Plasmin activity was measured by incubating samples in $100 \mathrm{mM}$ Tris buffer, pH 8.0, 0.13 M dithiobis, $20 \mathrm{mM}$ Z-Lys-S-Benzyl (Enzyme Systems Products, Livermore, CA). Stock solutions of Z-Lys-SBzl were prepared in distilled water. The rate of hydrolysis of Z-Lys-SBzl was measured spectrophotometrically by allowing the released benzyl mercaptan to react directly with dithiobis. The increase in absorbance at $412 \mathrm{~nm}$ due to formation of the carboxy-nitrophenoxide was measured three times, the average value was calculated, and the rate of change in absorbance was determined.

\section{Ulcer assessment}

All ulcers were photographed on admission and at each wound collection time point to provide visual record of any changes in appearance of the ulcer and healing rate. The reduction in surface area of all ulcers was measured by planimetry after 8 weeks (Pharma Med Concept, Düren, Germany).

\section{Statistical analysis}

Statistical analysis was performed using ANOVA and Bonferroni's post hoc test. Results were considered significant at a $P$-value below $0 \cdot 05$.

\section{Results}

\section{Elastase activity}

The wound fluid of patients treated by ORC/collagen matrix showed a significant reduction in elastase activity at day 5 and at all later time points compared with initial elastase activity measured at day 0. Furthermore, elastase activity was significantly decreased in patients treated with ORC/collagen matrix compared with the control group at days 5 and 14 (Figure 1).

\section{Plasmin activity}

Plasmin activity was significantly reduced at days 5 and 14 in comparison with control wounds (*). In addition, plasmin was significantly reduced on days 5, 14, 28 and 42 in patients treated with ORC/collagen matrix in comparison with the baseline parameters of plasmin activity in the treatment group (\#). At days 42 and 56, the plasmin activity in the control and treatment groups was almost equal, yet still reduced in comparison with baseline values (Figure 2).

\section{Healing rate}

After 12 weeks, wounds treated with ORC/collagen matrix showed a significant reduction in wound surface area by $65 \%$ versus $41 \%$ in the wounds of control group patients (Figure 3 ).

\section{Discussion}

In this study, we have measured the effect of an ORC/collagen matrix treatment on protease activity in wound fluid derived from pressure sores and compared this effect with a control treatment. We have shown that wound fluid from pressure sores contains high levels of elastase and plasmin activity, which can be reduced by treatment with ORC/collagen matrix. Furthermore, we have shown that treatment with ORC/collagen matrix leads to faster healing of pressure sores.

High protease activity may result due to one or more of the following: an increase in the expression of the protease, an increase in the extracellular activation of latent proteases or a reduction in the level of endogenous protease inhibitors. If extracellular proteolysis is excessive or poorly regulated, 


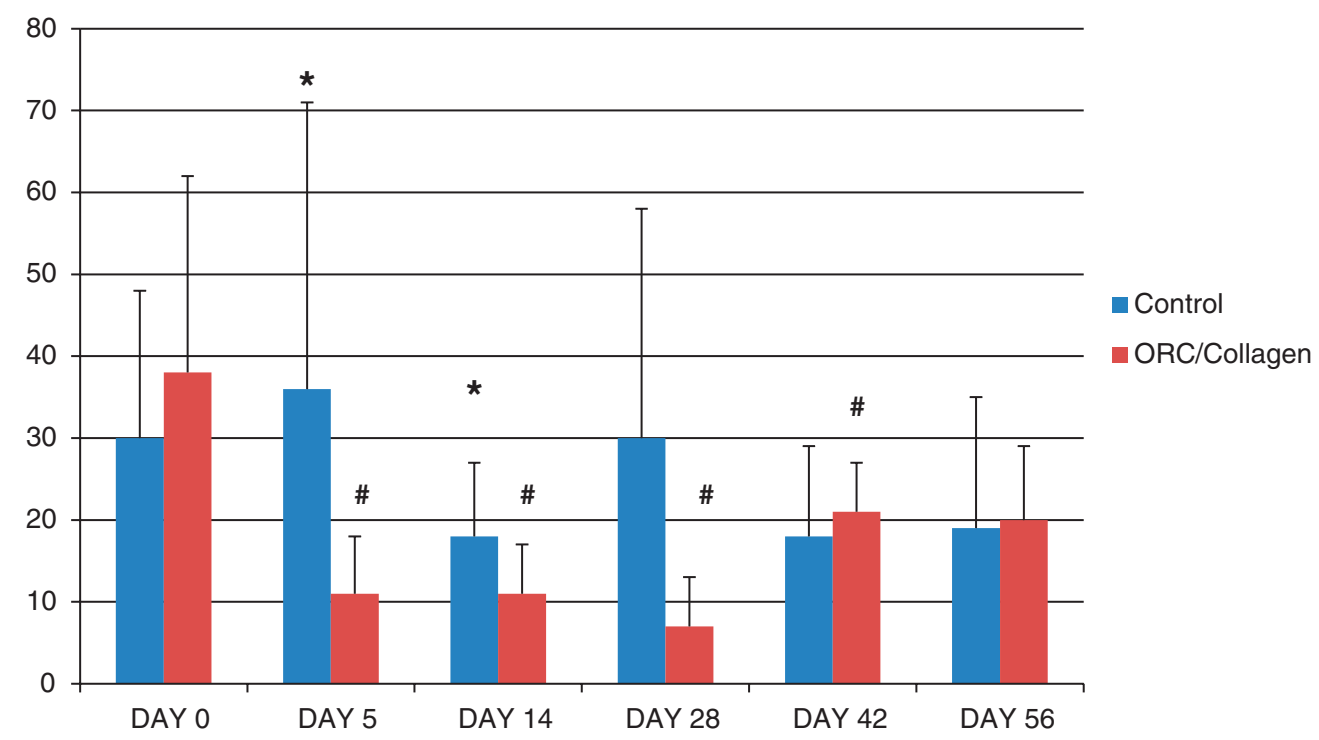

Figure 2 Activity of plasmin at different time points ( $y$-axis: $\mu \mathrm{U} / \mathrm{ml} / \mathrm{mg}$ protein). Plasmin activity was significantly reduced on days 5 and 14 versus control wounds. In addition, the plasmin activity was significantly reduced on days 5, 14, 28 and 42 in comparison with the baseline parameters of plasmin activity in the treatment group $(P<0.05, n=33)$.

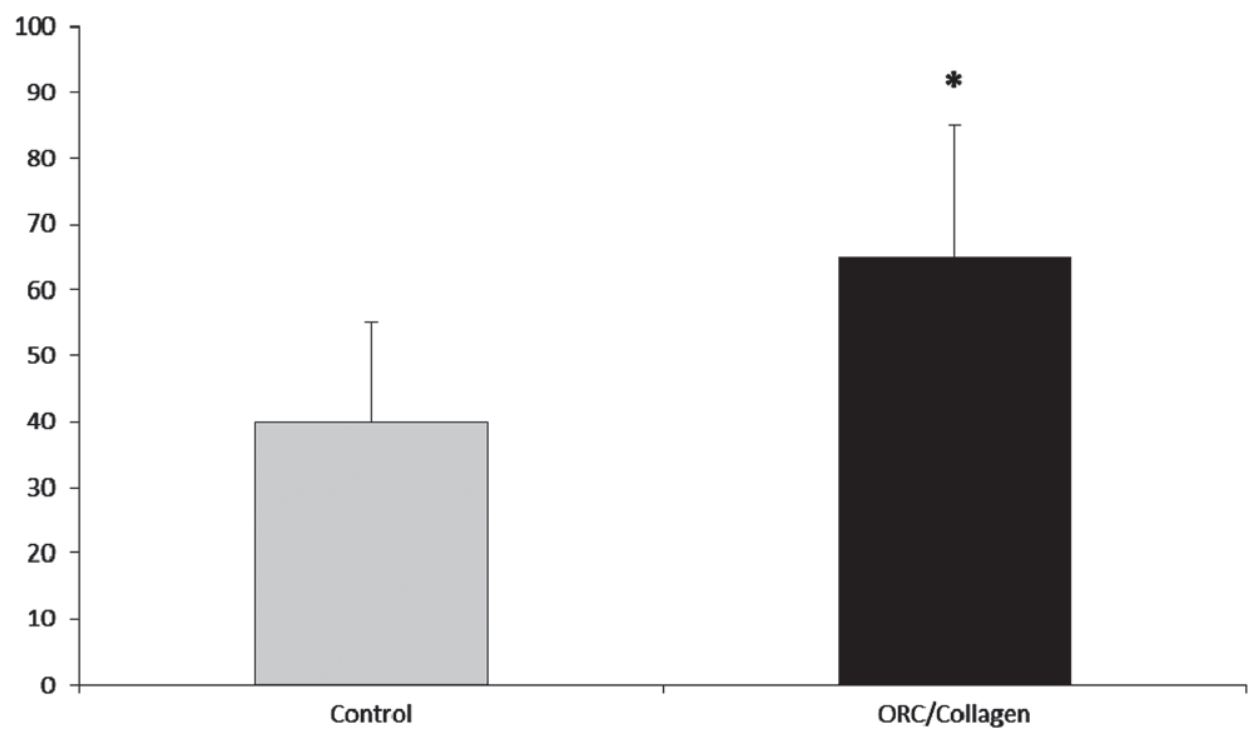

Figure 3 The wound surface area was significantly reduced $(P<0.05)$ in the oxidised regenerated cellulose $(O R C) /$ collagen group after 8 weeks $(y$-axis: percentage wound surface reduction) when compared with the control wounds ( $65 \% \pm 13$ versus $41 \% \pm 11, n=33$ ).

it can lead to generalised tissue destruction, a characteristic of many pathological inflammatory conditions including non-healing chronic wounds (23). This hypothesis is supported by several studies in which human chronic wound tissue and fluids have been analysed $(24,25)$. The combined use of collagen and oxidised regenerated cellulose has been shown to specifically inhibit the action of these proteases without affecting the activity of endogenous growth factor (14). Our study provides additional support to this mechanism as we found a significantly decreased activity of elastase and plasmin after 5 days of treatment with ORC/collagen matrix.
According to recent studies, high elastase activity may lead to degradation of fibronectin (26) and growth factors (6) in chronic wounds. This implies that these wounds are still in the inflammatory phase of wound repair even though all wounds had been present for at least 30 days.

Plasmin is the major fibrinolytic enzyme. It is possible that reduced plasmin activity in chronic wounds could contribute to fibrosis, which often occurs in leg ulcers $(2,27)$. Previous studies suggested that plasmin activity is increased in chronic wound fluid (28). The relationship between elevated levels of plasmin and reduced levels of VEGF was shown by Lauer et al. (11). The results indicated that chronic wound 
fluid contains enzymatic activity, in particular serine proteases, which might affect VEGF stability (11). VEGF plays an important role in wound healing, particularly during angiogenesis (11). In our patient group treated with ORC/collagen matrix, we found an accelerated healing of the pressure sores. This may be supported by reduced plasmin activity and increased VEGF levels.

The mechanisms responsible for the increased generation of plasmin in the chronic wound environment remain unclear. Previous studies have demonstrated that epidermal keratinocytes can regulate plasmin activation through the expression of mediators critical for plasmin activation, including the urokinase-type plasminogen activator and its receptor (29).

In previous studies, ORC/collagen reduced the activity levels of elastase and plasmin. However, the results of these studies are limited as they did not investigate these effects in vivo. In our study, we used a Z-Lys-SBzl assay, which is very sensitive for plasmin studies (30).

Previous clinical trials with ORC/collagen matrix have shown accelerated healing in venous leg ulcers and diabetic foot ulcers (14-21,31). In our present study, we observed a similar clinical effect in pressure sores.

Other advantages found when using ORC/collagen matrix include its ease of application. As the product is bio-absorbable, there was no need to remove the product at dressing changes. Furthermore, there was no infection observed in the ORC/collagen matrix group.

In summary, we have shown that ORC/collagen matrix treatment of pressure sores significantly reduces elastase and plasmin activity in wound exudates, thereby rebalancing the wound microenvironment and significantly improving the rate of healing.

\section{Acknowledgements}

The study was funded by an unrestricted research grant from Johnson \& Johnson Wound Management. The authors declare no conflict of interest.

\section{References}

1. Li W, Chong S, Huang E, Tuan T. Plasminogen activator/plasmin system: a major player in wound healing? Wound Rep Regen 2003;11:239-47.

2. Mustoe TA, O'Shaughnessy K, Kloeters O. Chronic wound pathogenesis and current treatment strategies: a unifying hypothesis. Plast Reconstr Surg 2006;117:35S-41S.

3. Lazarus GS, Cooper DM, Knighton DR, Margolis DJ, Pecorana RE, Rodeheaver G, Robson MC. Definitions and guidelines for assessment of wounds and evaluation of healing. Arch Dermatol 1994;130:489-93.

4. Nwomeh BC, Yager DR, Cohen IK. Physiology of the chronic wound. Clin Plast Surg 1998;25:341-56.

5. Agren MS, Eaglstein WH, Ferguson MW, Harding KG, Moore K, Saarialho-Kere UK, Schultz GS. Causes and effects of the chronic inflammation in venous leg ulcers. Acta Derm Venereol 2000;210:3-17.

6. Yager DR, Chen SM, Ward SI, Olutye OO, Diegelmann RF, Cohen IK. Ability of chronic wound fluids to degrade peptide growth factors is associated with increased levels of elastase activity and diminished levels of proteinase inhibitors. Wound Repair Regen 1997;5:23-32.

7. Owen CA, Campbell EJ. The cell biology of leukocyte-mediated proteolysis. J Leukoc Biol 1999;65:137-50.

8. Chapman HA. Plasminogen activators, integrins, and the coordinated regulation of cell adhesion and migration. Curr Opin Cell Biol 1997;9:714-24.

9. Parks WC, Mecham RP. Matrix metalloproteinases. San Diego: Academic Press, 1998.

10. Rifkin DB, Gleizes PE, Harpel J, Nunes I, Munger J, Mazzieri R, Noguera I. Plasminogen/plasminogen activator and growth factor activation. Ciba Found Symp 1997;212:105-15.

11. Lauer G, Sollberg S, Cole M, Flamme I, Stuerzebecher J, Mann K, Krieg T, Eming S. Expression and proteolysis of vascular endothelial growth factor is increased in chronic wounds. J Invest Dermatol 2000; 115:1-12

12. Hoffmann R, Starkey S, Coad J. Wound fluid from venous leg ulcers degrades plasminogen and reduces plasmin generation by keratinocytes. J Invest Dermatol 1998;111:1140-4.

13. Grinell F, Zhu M. Fibronectin degradation in chronic wounds depends on the relative levels of elastase, $\alpha 1$-proteinase inhibitor, and a2-macroglobulin. J Invest Dermatol 1996;106:335-41.

14. Cullen B, Smith R, McCulloch E, Silcock D, Morrison L. Mechanism of action of PROMOGRAN, a protease modulatingmatrix, for the treatment of diabetic footulcers. Wound Repair Regen 2002;10: $16-25$.

15. Ghatnekar O, Willis M, Persson U. Costeffectiveness of treating deep diabetic foot ulcers with Promogran in four European countries. $J$ Wound Care 2002;11:70-4.

16. Ulrich D, Smeets R, Unglaub F, Wöltje M, Pallua N. Effect of oxidized regenerated cellulose/collagen matrix on proteases in wound exudate of patients with diabetic foot ulcers. $J$ Wound Ostomy Continence Nurs 2011;38:522-8.

17. Motzkau M, Tautenhahn J, Lehnert H, Lobmann R. Expression of matrix-metalloproteases in the fluid of chronic diabetic foot wounds treated with a protease absorbent dressing. Exp Clin Endocrinol Diabetes 2011;119:286-90.

18. Lobmann R, Zemlin C, Motzkau M, Reschke K, Lehnert H. Expression of matrix metalloproteinases and growth factors in diabetic foot wounds treated with a protease absorbent dressing. J Diabetes Complications 2006;20:329-35.

19. Gottrup F, Cullen BM, Karlsmark T, Bischoff-Mikkelsen M, Nisbet L, Gibson MC. Randomized controlled trial on collagen/oxidized regenerated cellulose/silver treatment. Wound Repair Regen 2013;21:216-25.

20. Vin F, Teot L, Meaume S. The healing properties of Promogran in venous leg ulcers. $J$ Wound Care 2002;11:335-41.

21. Smeets R, Ulrich D, Unglaub F, Wöltje M, Pallua N. Effect of oxidised regenerated cellulose/collagen matrix on proteases in wound exudate of patients with chronic venous ulceration. Int Wound $J$ 2008;5:195-203.

22. Bradford MM. A refined and sensitive method for the quantitation of microgram quantities of protein utilising the principle of protein-dye binding. Anal Biochem 1976;72:248-54.

23. Vaheri A, Stephens RW, Salonen EM, Pollanen J, Tapiovaara H. Plasminogen activation at the cell surface-matrix interface. Cell Differ Dev 1990;32:255-62.

24. Bullen EC, Longaker MT, Updike DL, Benton R, Ladin D, Hou Z, Howard EW. Tissue inhibitor of metalloproteinases-1 is decreased and activated gelatinases are increased in chronic wounds. $J$ Invest Dermatol 1995;104:236-40.

25. Parks WC. Matrix metalloproteinases in repair. Wound Repair Regen 1999;7:423-32.

26. Rao CN, Ladin DA, Liu YY, Chilukuri K, Hou ZZ, Woodley DT. $\alpha 1$-Antitrypsin is degrade and non-functional in chronic wounds but intact and functional in acute wounds: the inhibitor protects fibronectin from degradation by chronic wound fluid enzymes. J Invest Dermatol 1995:105:572-8. 
27. Falanga V. Chronic wounds: pathophysiologic and experimental considerations. J Invest Dermatol 1993;100:721-5.

28. Palolahti M, Lauharanta J, Stephens RW, Kuusela P, Vaheri A. Proteolytic activity in leg ulcer exudate. Exp Dermatol 1993;2:29-37.

29. Schäfer BM, Stark HJ, Fusenig NE, Todd RF, Kramer MD. Differential expression of uPA, ist receptor (uPA-R), and inhibitor type-2 (PAI-2) during differentiation of keratinocytes in an organotypic coculture system. Exp Cell Res 1995;220:415-23.
30. Green G, Shaw E. Thiobenzyl benzyloxycarbonyl-L-lysinate, substrate for a sensitive colorimetric assay for trysin-like enzymes. Anal Biochem 1979;93:223-6.

31. Veves A, Sheehan P, Pham HT. A randomized, controlled trial of Promogran (a collagen/oxidized regenerated cellulose dressing) vs standard treatment in the management of diabetic foot ulcers. Arch Surg 2002;137:822-7. 